
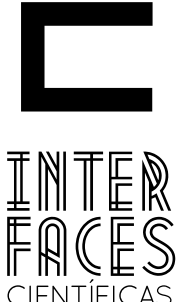

SAÚDE E AMBIENTE

\title{
PERFIL DOS ATENDIMENTOS PSIQUIÁTRICOS REALIZADOS PELO SERVIÇO DE ATENDIMENTO MÓVEL DE URGÊNCIA, ARACAJU, JANEIRO/2010 A FEVEREIRO/2011
}

\section{RESUMO}

Manuela de C. Vieira Martins

Lidiane Vieira Barbosa ${ }^{3}$

Simone Alves Garcez Guedes

\author{
Vanessa Tavares de Gois Santos \\ Cintia Regina Guerra Corrêa ${ }^{4}$
}

A Política Nacional de Urgência e Emergência garante atenção integral ao atendimento das urgências clínicas, cirúrgicas, gineco-obstétricas, psiquiátricas, pediátricas e as relacionadas às causas externas. O SAMU-192, que foi instituído através da Portaria 1.864/03, é o serviço responsável por atender todos os chamados de urgência e emergência da população. A crise psiquiátrica é vista como uma urgência, visto que o usuário necessita de atendimento o mais rápido possível para evitar possíveis danos a si mesmo ou de outras pessoas. Por conta disso objetivou-se caracterizar os atendimentos psiquiátricos realizados pelo SAMU Aracaju, no período de janeiro/2010 a fevereiro/2011. A pesquisa foi realizada no banco de dados do SAMU Aracaju. Em relação ao gênero, verificou-se que $69 \%$ foram do sexo masculino e os principais motivos das ocorrências foram classificados como "agitado" (51\%) e transferências (23\%). 0 principal destino final dos atendimentos foi a Urgência Psiquiátrica do município (59,3\%). Enfim, pôde-se concluir que o SAMU Aracaju é um importante meio para o atendimento das urgências psiquiátricas, por isso, é importante que gestores realizem constantemente o monitoramento desses atendimentos visando planejar ações intersetoriais que contribuam para a otimização de todos os serviços de saúde e, consequentemente, gerem atendimentos de qualidade a toda população.

\section{PALAVRAS-CHAVE}

Serviços Médicos de Emergência. Psiquiatria. Medicina de Emergência. 


\section{ABSTRACT}

The National Emergency Policy guarantees that the urgent medical, surgical, gynecological, obstetric, psychiatric, and pediatric situations, as well as other ones related to external causes may be assisted. SAMU-192, which was established by Decree $1.864 / 03$, is the department which is responsible for answering all urgent calls concerning the health care of the population. The psychiatric crisis is seen as an emergency, since the user needs quick service in order to avoid that this person gets hurt or even hurts someone else. Because of this, the psychiatric care, performed by SAMU Aracaju, from January/2010 to February/2011 was characterized. The survey was conducted in the database of SAMU Aracaju. Regarding gender, it was found that $69 \%$ were male and the main occurrences were classified as "busy" (51\%) and transfers $(23 \%)$. The main destination of the patients was the Psychiatric Emergency of the city (59.3\%). It can be concluded that the SAMU Aracaju is an important means to assist the psychiatric emergency, so it is important that managers constantly monitor these calls seeking intersectoral planning that may contribute to the optimization of all health services and therefore generate good health care to the entire population.

\section{KEYWORDS}

Emergency Medical Services. Psychiatry. Emergency Medicine.

\section{INTRODUÇ̃̃O}

A Portaria $n^{0} 1.863 / 03$ instituiu a Política Nacional de Atenção às Urgências, garantindo atenção integral ao atendimento das urgências clínicas, cirúrgicas, gineco-obstétricas, psiquiátricas, pediátricas e as relacionadas

\section{RESUMEN}

La política Nacional de Urgencia y Emergencia garantiza la atención integral para atender la emergencia clínica, quirúrgica, ginecológica, obstétrica, psiquiatría, pediatría y por causas externas. El SAMU-192, que fue establecido por el Decreto 1.864/03, es el departamento encargado de responder a todas las convocatorias de la población de atención de urgencia y de emergencia. La crisis psiquiátrica es vista como una emergencia, ya que el usuario necesita atención tan pronto como sea posible para evitar un posible daño a sí mismo o a otros. Por eso, se quiso caracterizar la atención psiquiátrica realizada por el SAMU de Aracaju, en el período de Enero/2010 Febrero/2011. La investigación se realizó en la base de datos del SAMU Aracaju. En cuanto al género, se encontró que el $69 \%$ eran hombres y las razones principales de los sucesos fueron clasificados como "ocupado" (51\%) y las transferencias (23\%). El principal destino de las visitas fue el hospital de Emergencias Psiquiátricas en la ciudad (59,3\%). De todos modos, se concluyó que la SAMU Aracaju es un medio importante para atender la emergencia psiquiátrica, por lo que es importante que los directivos realicen un seguimiento constante de estas llamadas en busca de la planificación intersectorial que contribuyan a la optimización de todos los servicios de salud y por lo tanto, generar una atención de calidad a toda la población.

\section{PALABRAS CLAVE}

Servicios Médicos de Urgencia. Psiquiatría. Medicina de Emergencia. 
O SAMU é responsável por atender aos chamados de urgência e emergência de toda a população. Esses atendimentos podem ocorrer em via pública, em um estabelecimento de saúde e até mesmo nas residências (CABRAL; SOUZA, 2008).

A urgência em saúde mental é definida quando o sujeito em crise necessita de um atendimento rápido visando uma resposta positiva da crise e que esta consiga evitar danos à integridade física e psíquica no futuro (BONFADA, 2010).

Como o SAMU funciona através de um serviço de regulação médica e tem acesso fácil ao local do atendimento, a maioria dos casos de urgência psiquiátrica é resolvida por esse Serviço, uma vez que ele consegue atender a crise com agilidade e garantindo encaminhamentos mais eficazes (BONFADA, 2010).

\section{METODOLOGIA}

Trata-se de um estudo descritivo, baseado na coleta das informações das 2919 fichas de atendimento psiquiátrico realizados pelo SAMU-192 Aracaju no período de janeiro/2010 a fevereiro/2011. Essas fichas são preenchidas e registradas pelos Técnicos Auxiliares de Regulação Médica (TARM) e médicos reguladores a cada ocorrência. Posteriormente, são arquivadas em um software na própria sede do serviço, compondo o banco de dados do SAMU-192 Aracaju.

Os dados contidos nas fichas eletrônicas foram analisados em cinco variáveis: gênero, endereço da ocorrência, destino final, mês e motivo da solicitação. Apenas essas variáveis foram escolhidas, pois todas as outras - número da ocorrência, nome do solicitante, nome da vítima e telefone do solicitante - não constituem informações importantes e necessárias para garantir o objetivo da pesquisa. Para
Além de contribuir diretamente com os atendimentos da sociedade, o SAMU também é visto como observatório da situação de saúde da população, podendo - muitas vezes - mostrar o grau de resolubilidade dos serviços de saúde. Ele é capaz de identificar os problemas de saúde da população, fornecendo dados epidemiológicos à gestão para que esta possa atuar nas principais necessidades de saúde (AQUINO, 2007).

Cabral (2007) ainda relata que a análise dessas informações do SAMU é fundamental, pois, ela vai subsidiar o planejamento de ações visando melhorar o atendimento de saúde da população. Por conta disso, objetivou-se caracterizar os atendimentos psiquiátricos realizados pelo SAMU Aracaju no período de janeiro/2010 a fevereiro/2011.

essa análise foi considerado o valor de alfa como 0,05 e os dados foram mensurados através do programa Epi info.

O tratamento e análise dos dados foram realizados de forma descritiva, com distribuição absoluta das informações coletadas e apresentados sob forma de figuras.

0 presente estudo recebeu autorização do Comitê de Ética em Pesquisa da Universidade Tiradentes, obedecendo às recomendações da Resolução 196/96 do Conselho Nacional de Saúde, como também do Núcleo de Educação Permanente em Saúde de Aracaju (setor responsável pelas pesquisas realizadas em qualquer setor da Secretaria de Saúde de Aracaju). 


\section{RESULTADOS}

Duas mil e novecentos e vinte e nove (2929) fichas eletrônicas foram incluídas na pesquisa, relativas ao número total de atendimento psiquiátrico realizado pelo SAMU Aracaju de janeiro de 2010 a fevereiro de 2011. Destas, foram excluídas 10 fichas por se tratarem de informações de aten- dimentos duplicados, o que totalizou 2919 fichas analisadas.

Os principais motivos de solicitação do atendimento do SAMU foram por causa de usuários agitados (51\%), seguido das transferências (23\%) (Figura 1).

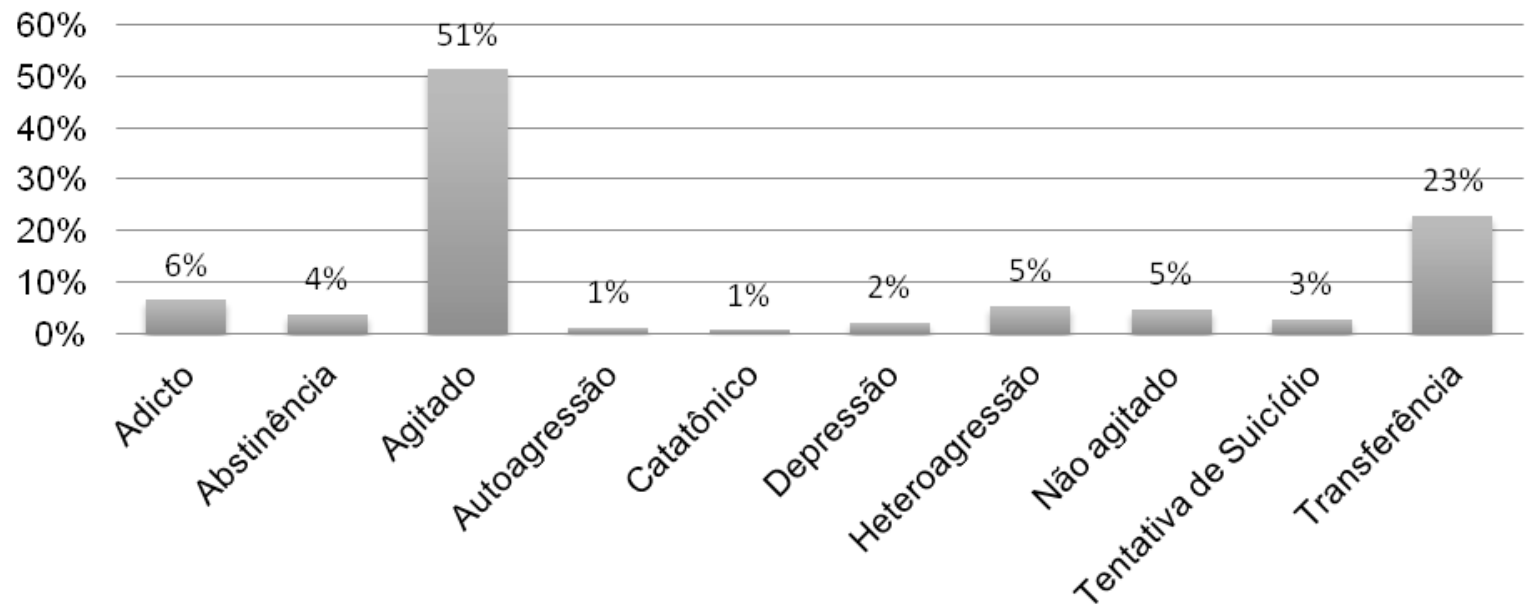

Figura1: Motivos dos atendimentos psiquiátricos do SAMU Aracaju, janeiro/2010 à fevereiro/2011.

Quanto ao gênero, o maior número de atendimentos foi realizado à população masculina $(69 \%)$. As mulheres corresponderam a $30 \%$ dos atendimentos, restando $1 \%$ para as amostras em que não foi possível essa identificação. Em relação à origem dos atendimentos, que foi dividido e identificado através do bairro, a distribuição foi bem homogênea. Porém, os bairros de maior destaque foram: Santo Antônio (13,9\%), 18 do Forte $(7,1 \%)$ - ambos na Zona Norte da capital sergipana - São Conrado (8,8\%) e Santa Maria (6\%) ambos na Zona Sul (Figura 2). Vale enfatizar, também, a alta taxa de ocorrências sem identificação do local de origem (11,2\%).
Quanto ao destino final dos atendimentos, mais da metade $(59,3 \%)$ foi regulado para a Urgência Psiquiátrica do Hospital São José, deixando um percentual menor para outros hospitais do município, a exemplo do Hospital Dr. Nestor Piva (Zona Norte) com 16,9\% e do Hospital Fernando Franco (Zona Sul) com 4,7\% (Figura 3). Em relação ao período do ano, observou-se grande homogeneidade em relação aos meses, variando de 5\% (Janeiro/2011 e Fevereiro/2011) a 9\% (Dezembro/2010) (Figura 4). 


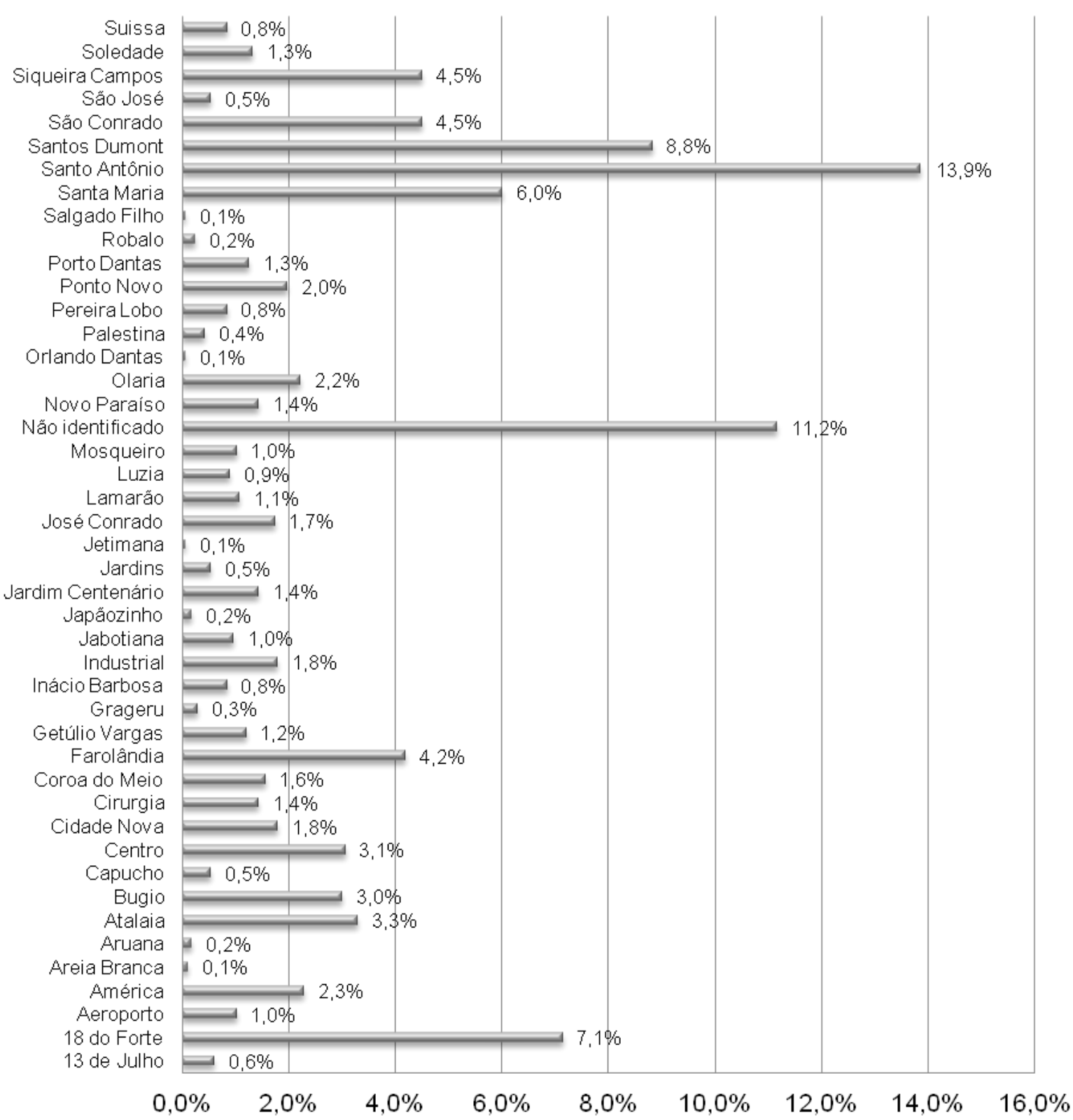

Figura 2: Origem dos atendimentos psiquiátricos do SAMU Aracaju, segundo bairro, janeiro/2010 à fevereiro/2011. 
Clínica de Repouso São Marcelo Hospital São Lucas

Urgência Mental Hosp. São José

Clínica Psiq. Santa Maria

Clínica e Maternidade Santa Helena Residência Terapéutica Hospital Renascença Pronto Clínica Hospital Primavera

Pediatria Hospital Santa Izabel

PAIPES

Não identificado

Hospital Dr. Nestor Piva

Hospital Fernando Franco

Hospital de Urgência de Sergipe Hospital Universitário Hospital da Polícia Militar

Hospital Cirurgia

CAPS III

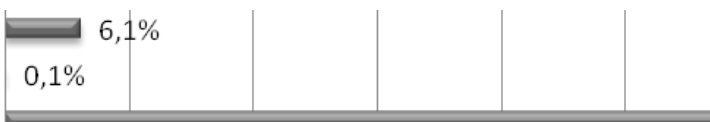

$=$

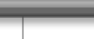

$8,9 \%$

$0,1 \%$

$0,1 \%$

$0,1 \%$

$0,1 \%$

$0,1 \%$

$0,1 \%$

$0,1 \%$

$0,8 \%$

$4,7 \%$

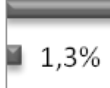

$0,1 \%$

$0,1 \%$

$0,1 \%$

- $1,1 \%$

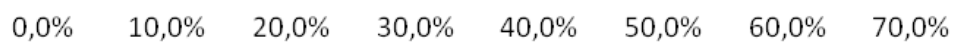

Figura 3: Destino final dos atendmentos psiquiátricos realizados pelo SAMU Aracaju, janeiro/2010 à fevereiro/2011.

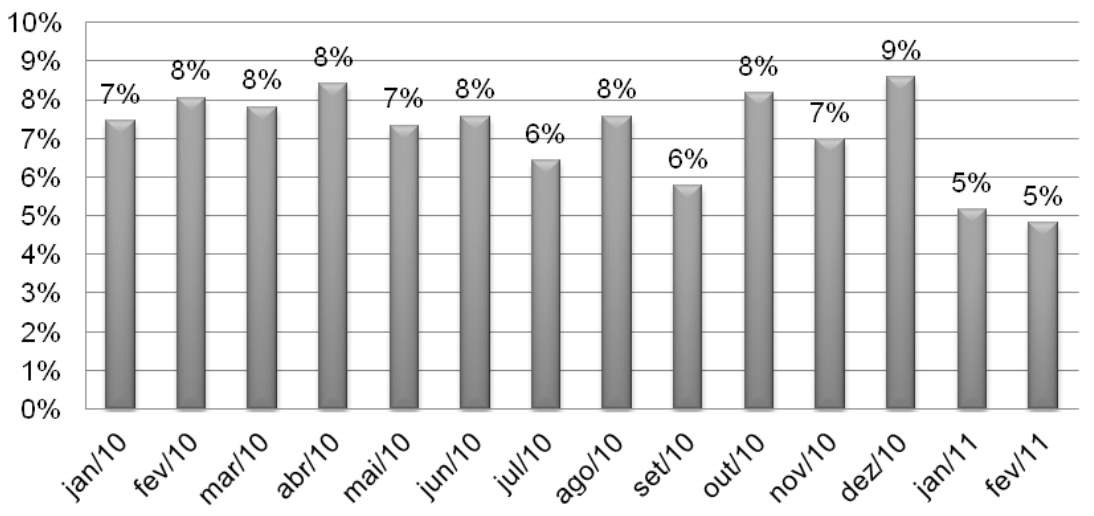

Figura 4: Percentual dos atendimentos psiquiátricos realizados pelo SAMU Aracaju, segundo mês, janeiro/2010 à fevereiro/2011. 


\section{DISCUSSÃO}

O SAMU Aracaju é responsável por inúmeras ocorrências anualmente, desde a sua implantação em 2002. Contudo, os atendimentos psiquiátricos se destacam a cada ano. Em sua dissertação de mestrado, Jardim (2008) observou que no ano de 2004 ocorreram 2.584 atendimentos psiquiátricos, o que corresponde a $6,7 \%$ do total de atendimentos. Esse número foi aumentando no decorrer dos anos, chegando a 2.785 (8,2\%) em 2005 e 3.408 (8,9\%) em 2006.

0 estudo de Pitteri e Monteiro (2010) constatou que o principal motivo de solicitação de viatura do SAMU a pacientes psiquiátricos é a agitação, resultado igualmente encontrado nesta pesquisa. Para Oliveira, Oliveira e Machado (2009), a agitação psicomotora é manifestada quando há abuso de algumas substâncias tóxicas (Ex: drogas) ou acarretada por alguma outra condição. Por conta disso, o paciente pode tornar-se violento e agressivo, gerando então uma situação de urgência psiquiátrica.

Em relação às transferências, outro motivo evidente nas solicitações do SAMU Aracaju, Moreira et al. (2011) relatam que elas fazem parte dos serviços e redes de saúde de qualquer local. Para os autores, o ato de transferir uma vítima para outro estabelecimento consiste no fato da busca por atendimento mais especializado, que possibilite atender as necessidades do paciente.

Ao falar do gênero da vítima do atendimento psiquiátrico no SAMU, alguns estudos demonstraram que os homens constituem as maiores vítimas (PITTERI; MONTEIRO, 2010; MICHELS; ARCOVERDE, 2012). Apesar de no Brasil a relação homem-mulher com algum tipo de transtorno mental ser 1:2 (PITTERI; MONTEIRO, 2010), essa estatística no SAMU pode ser explicada pela hipótese de que as mulheres têm uma maior/melhor adesão ao tratamento de sua doença nos Centros de Atenção Psicossocial (CAPS), fazendo com que os homens iniciem uma crise psiquiátrica mais facilmente (VOLPE et al., 2010).

0 alto índice de solicitações em alguns bairros do município de Aracaju pode estar relacionado ao aspecto populacional, ou seja, geograficamente são os bairros com maior contingente populacional. Contudo, o percentual de solicitação "não identificada" também chama a atenção, fato também encontrado no estudo de Duarte et al., 2011. Para Sousa (2010), o levantamento dos dados das ocorrências do SAMU constitui informações fundamentais na área da saúde, pois, são capazes de auxiliar no planejamento de ações, recursos humanos e materiais.

A respeito do destino final das vítimas atendidas pelo SAMU Aracaju, o elevado percentual dos pacientes encaminhados à Urgência Mental do Hospital São José é evidenciado por esse serviço ser a única urgência psiquiátrica da capital. Jardim (2008) comenta que a Urgência Mental São José foi inaugurada em 2005, porém suas atividades se iniciaram apenas no início de 2006. A partir daí, a Urgência Mental passou a receber uma média de $7,5 \%$ das ocorrências psiquiátricas reguladas pelo SAMU Aracaju.

Em relação à distribuição temporal das ocorrências psiquiátricas, Sousa (2010) encontrou em seu estudo que os meses com maior percentual de atendimento foram: agosto, setembro e novembro. E os três primeiros meses do ano são aqueles em que o percentual é menor. Michels e Arcoverde (2012) também encontraram informações parecidas, pois, os meses de outubro, novembro e dezembro foram aqueles com maior taxa de atendimento, e os três meses do primeiro semestre (janeiro, fevereiro e abril) com menor taxa. Apesar de não encontrar nenhuma explicação para isso na literatura, esse fato pode estar relacionado ao período das festividades de final de ano, em que muitos usuários ultrapassam o limite de uso de substâncias psicoativas, principalmente o álcool. 


\section{CONCLUSÃO}

Observou-se, no período da pesquisa, que os principais atendimentos psiquiátricos realizados pelo SAMU Aracaju foram a usuários com algum tipo de agitação psicomotora, seguido das transferências. Assim como mostram outros estudos, os resultados da pesquisa em questão demonstram que os homens lideram a lista das urgências psiquiátricas.

\section{REFERÊNCIAS}

AQUINO, D.I. Caracterização do Serviço de Atendimento Móvel de Urgência (SAMU-192), do município de Florianópolis/SC. Dissertação de mestrado em Saúde e Gestão do Trabalho, Centro de Educação de Ciências da Saúde da Universidade do Vale do Itajaí. Itajaí, 2007.

BONFADA, D. Serviço de Atendimento Móvel de Urgência (SAMU) e a assistência às urgências psiquiátricas. Dissertação de Mestrado em Enfermagem. Universidade Federal do Rio Grande do Norte, Natal, 2010.

BRASIL (2003). Portaria 1.863/GM de 29 de setembro de 2003. Institui a Política Nacional de Atenção às Urgências e Emergências, a ser implantada em todas as unidades federadas, respeitadas as competências das três esferas de gestão. Brasília: Ministério da Saúde.

BRASIL (2003). Portaria 1.864/GM de 29 de setembro de 2003. Institui o componente pré-hospitalar móvel da Política Nacional de Atenção às Urgências, por intermédio da implantação de Serviços de Atendimento Móvel de Urgência em municípios e regiões de todo território brasileiro: SAMU-192. Brasília: Ministério da Saúde.
Em relação à situação geográfica dos atendimentos pode-se enfatizar o alto percentual de locais não identificados, caracterizando uma falha no primeiro atendimento do SAMU. Sobre o destino final, como era esperado, a Urgência Mental Hospital São José caracterizou-se como o principal local de acolhimento do paciente em crise, o que se justifica por se tratar da única referência de urgência psiquiátrica na capital.

CABRAL, A.P.S. Um termômetro do Sistema Único de Saúde - O Serviço de Atendimento Móvel de Urgência (SAMU-192): Análise do Modelo em uma Cidade do Nordeste Brasileiro. Recife: Fundação Oswaldo Cruz, 2007.

CABRAL, A.P.S.; SOUZA, W.V. Serviço de Atendimento Móvel de Urgência (SAMU): análise da demanda e sua distribuição espacial em uma cidade do Nordeste Brasileiro. Rev Bras Epidemiologia. v.11, n.4; p. 530 540, 2008.

DUARTE, S.J.H.; LUCENA, B.B.; MORITA, L.H.M. Atendimentos prestados pelo serviço móvel de urgência em Cuiabá, MT, Brasil. Rev. Eletr. Enf. jul./set. 2011.

JARDIM, K.F.S.B. 0 serviço ambulatorial móvel de urgência (SAMU) no contexto da reforma psiquiátrica: em análise a experiência de Aracaju/SE. Dissertação de mestrado em Psicologia, Universidade Federal do Rio Grande do Norte. Natal, 2008.

MICHELS, N.A.; ARCOVERDE, M.A.M. Perfil dos casos psiquiátricos atendidos em um serviço de atendimento móvel de urgência (SAMU). In: VII Seminário de Ensino, Pesquisa e Extensão do Centro de Educação e Letras da Universidade Estadual do Oeste do Paraná, Campos Foz do Iguaçu. Anais do VII SEPECEL, 2012. 
MOREIRA, F.M. et al. Serviços de Urgência e Emergência - 192: incidência dos atendimentos em um município do Vale do Paraíba. Emergência clínica, 2011.

OLIVEIRA, I.B.; OLIVEIRA, A.B.A.; MACHADO, I.C.S. Atendimento as pacientes psiquiátricos atendidos pelo SAMU 192, em Vitória da Conquista - BA. Diálogos \& Ciência. Ano III, n. 9, jun., 2009.

PITTERI, J.S.M.; MONTEIRO, P.S. Caracterização do Serviço de Atendimento Móvel de Urgência (SAMU) em Palmas - Tocantins, Brasil, em 2009. Com. Ciências Saúde, 2010.

Recebido em: 29 de junho de 2012 Avaliado em: 1 de agosto de 2012 Aceito em: 11 de agosto de 2012
SOUSA, W.R. Levantamento das ocorrências atendidas pelo Serviço de Atendimento Móvel de Urgência - SAMU no Município de Cuiabá/MT e Baixada Cuiabana no ano de 2009. Emergência clínica, 2010.

VOLPE, F.M. et al. Perfil da clientela atendida em um serviço público de urgência psiquiátrica no município de Belo Horizonte, Brasil, no período de 2002 a 2007. J Bras Psiquiatr, 2010. J Bras Psiquiatr. v. 59, n. 3, p. 203 - 209, 2010.

1 Enfermeira, Residente em Saúde Coletiva pela Universidade Tiradentes UNIT. Aracaju, Sergipe, Brasil. E-mail: manuela.cvm@hotmail.com

2 Cirurgiã dentista, Residente em Saúde Coletiva pela Universidade Tiradentes - UNIT. Aracaju, Sergipe, Brasil. E-mail: vanodonto@hotmail.com

3 Assistente social, Residente em Saúde Coletiva pela Universidade Tiradentes - UNIT. Aracaju, Sergipe, Brasil. E-mail: lidiane.vieira21@hotmail. com

4 Enfermeira do Serviço de Atendimento Móvel de Urgência de Aracaju, Especialista em Saúde Pública. Aracaju, Sergipe, Brasil. E-mail: cintiacuca@hotmail.com

5 Cirurgiã dentista, Coordenadora da Residência Multiprofissional Integrada em Saúde Coletiva da Universidade Tiradentes - UNIT, Mestre em Saúde e Ambiente. Aracaju, Sergipe, Brasil. E-mail: simonegguedes@ yahoo.com.br 